\title{
A Study of D2-40 Immunohistochemical Expression in Colorectal Carcinomas
}

\author{
Sarvek Bajaj*, Gururajaprasad. C and Suchitha S \\ Department of Pathology, JSS Medical college, JSS university, Mysore, India
}

\section{ABSTRACT}

Background: Colorectal cancer is one of the most common cancers. Factors affecting survival include nodal positivity, lymphovascular invasion (LVI) and lymphangiogenesis (role in metastasis). Most endothelial markers stain lymphatics and blood vessels without discrimination. Recent marker D2-40 is specific for lymphatic endothelium.

This study aims to interpret the utility of D2-40 in detecting LVI, which would be missed on H\&E; and in analysing lymphatic vessel density and LVI as predictive markers for the risk of nodal metastases in colorectal carcinomas.

Methods: Study was conducted on 40 specimens of colorectal carcinomas. Immunohistochemistry was performed with D2-40. Stastical analysis was by Spearman's correlation and Chi square test

Result: Mean age was 55.50 years. Of 40 cases, majority were in ascending colon followed by rectum, sigmoid, transverse and descending colon. $80.0 \%$ were adenocarcinomas, $15.0 \%$ mucinous adenocarcinomas and $5.0 \%$ signet-ring cell carcinomas. Tumors were moderately differentiated in $80.0 \%$ and poorly in $20.0 \%$. Most patients were in stage T3 followed by T2 and T4. Most common nodal stage was N0 followed by N1, N2 and N3.

LVI was detected in eleven more cases on D2-40 than H\&E and correlated with lymphatic density and nodal status. Lymphatic density correlated with nodal status and pT. Peritumoral lymphatics were found in $95 \%$ cases and intratumoral lymphatics in $90 \%$. Intratumoral lymphatics correlated with nodal status.

Conclusion: D2-40 increased the detection rate of LVI as compared to H\&E and showed the value of lymphangiogenesis in disease progression and metastasis.

\section{Keywords: Colorectal Adenocarcinoma, D2-40 Immunohistochemistry, Lymphovascular Invasion, Lymphatic Density}

\section{Introduction}

Globally, nearly 800,000 new colorectal cancer cases occur each year (10\% of all cancers) ${ }^{[1]}$ Colorectal cancer (CRC) is the third commonest in men and second in women. ${ }^{[2]}$ Multiple prognostic factors affect the survival of patients. The most important are metastases, local tumor extent, nodal positivity and status, lymphovascular invasion and the status of resected margins.

Lymph node metastases is an important prognostic indicator for disease progression and crucial for therapeutic strategies. Not much is known regarding the mechanisms through which tumor cells enter the lymphatic system. Some investigators have suggested that lymphangiogenesis plays an active role in metastasis.

The study of lymphangiogenesis, growth and production of new lymphatic vessels under several physiological and pathological conditions has gained more attention in the recent years. Presently, there is no consensus whether the major pathway of lymphatic spread is through the development of lymphatic vessels intra or peritumorally, with studies published supporting each of the possibilities

Lymphangiogenesis has been difficult to investigate because there was a lack of specific antibodies recognizing the lymphatic endothelium. Most available endothelial markers used to assess intratumoral micro vessel density, a reflection of tumor angiogenesis, stained both lymphatics and blood vessels without discrimination. [3] Recently, lymphatic endothelial specific markers have become available, which facilitate the analysis of lymphangiogenesis in cancer. ${ }^{[4]} \mathrm{D} 2-40$ (also known as antipodoplanin), an IgG2a monoclonal antibody, was generated against an oncofetal membrane antigen M2A and identified in ovarian carcinoma cell lines and germ cell neoplasia. ${ }^{[5]}$ It has shown to stain endothelium of lymphatic vessels and lymphangiomas but negative in hemangiomas. ${ }^{[6]}$

D2-40 highlights the lymphatics and outlines the tumor emboli otherwise indiscernible by hematoxylin and eosin stain and increases the detection rate of lymphatic invasion. 
[7] In the absence of effective immunohistochemical markers of lymphatic endothelium in paraffin sections, lymphatic invasion is identified on the basis of conventional hematoxylin and eosin (H\&E) staining. Pitfalls in the technique arise mainly from the difficulty in visualizing the lymphatic vessel wall following H\&E staining. ${ }^{[6]}$

\section{Aims and Objectives}

To assess the lymphatic microvessel density and lymphovascular invasion using D2-40 immunostaining in colorectal carcinomas

To evaluate the association of lymph vessel density (LVD) (peritumoral and intratumoral) and lymphovascular invasion with tumor size, stage (pT), grade and lymph node status.

To compare the detection of lymphovascular invasion (LVI) by hematoxylin and eosin staining with D2-40 immunostaining

\section{Materials and Methods}

Study Population: 40 patients with colorectal carcinoma were studied between September 2013 and Aug 2016 in the Department of Pathology, JSS Medical College, Mysore. The following data were reviewed: age, gender, tumor location, size, histological type, grade, TNM staging and LVI.

Type of Study: Descriptive study; One year of retrospective (September 2013 - August 2014) and 2 years of prospective study (September 2014 - August 2016)

Inclusion Criteria: Adenocarcinomas.

Exclusion Criteria: Other malignancies.

Histology and TNM Classification: Specimens were fixed in $10 \%$ of formalin for $24-48$ hours and embedded in paraffin. Deparaffinzed sections were stained with H\&E and classified according to WHO. American Joint Committee Of Cancer (AJCC), seventh edition, was followed for TNM staging.

Immunohistochemical Staining: The paraffin blocks of the tumor with maximum depth of invasion were selected. $4 \mu \mathrm{m}$ sections were taken on Poly-L-Lysine slides. After deparaffinizing and removing endogenous peroxidase, ready-to-use primary antibody ((DAKO Monoclonal Mouse Anti-D2-40, Code IS072) was applied and antigen retrieval was done using a pressure cooker followed by secondary antibody (DAKO). Subsequently DAB-chromogen was added. Slides were stained with hematoxylin and mounted with DPX. Tonsil was used as positive control. For negative controls, primary antibody was omitted.
Method of Reporting by Immunohistochemistry (IHC): Staining pattern of D2-40 is cytoplasmic and membranous and it stains only lymphatic endothelial cells. After scanning at low magnification $(\mathrm{x} 40)$, three areas with greatest number of distinct intratumoral lymphatic foci (hot spots) were selected by two observers and independently evaluated for microvessel (MV) counts using x400 magnification without knowing the patient status. In the absence of apparent hotspots, three or more randomly selected areas were counted. The highest number of lymphatic vessels counted was recorded and used for statistics. Intratumoral lymphatic vessels were defined as vessels within the main tumor mass, surrounded by tumor cells, with no RBCs in lumina. Vessels more than one high power field (HPF) away from the tumor front were considered as peritumoral lymphatic vessels. Single immunoreactive endothelial cells, or endothelial cell clusters separate from other MVs, were counted as a vessel. The highest number of vessels counted were recorded. LVD was classified into low ( $<10$ vessels / HPF), moderate (10-15 vessels / HPF) and high ( $>15$ vessels / HPF). Lymphovascular invasion was considered evident if at least one tumor cell cluster was clearly visible inside the vascular space. ${ }^{[4],[8]}$

Stastical Analysis: The association of clinicopathological data with LVD and LVI on D2-40 was evaluated using Chi-square test and Spearman's coefficient of correlation as appropriate. The difference between LVI on D2-40 and $\mathrm{H} \& \mathrm{E}$ was tested with McNemar test. Statistical analyses was performed with SPSS software 23. P $<0.05$ was considered statistically significant.

\section{Result}

40 patients were from 25-82 years with a mean of $55.50 \pm$ 14.122, maximum number (12) between 41-50 and least number (2) between 81-90 years. $23(57.5 \%)$ cases were males and $17(42.5 \%)$ were females with a male to female ratio of $1.35: 1$.

12 tumors $(30 \%)$ were located in the ascending colon, 05 $(12.5 \%)$ in transverse colon, $03(7.5 \%)$ in descending colon, $9(22.5 \%)$ in sigmoid colon, and $11(27.5 \%)$ in the rectum. Tumor size ranged from 2.5 to $11 \mathrm{~cm}$ with a mean of $5.67 \pm$ $2.37 \mathrm{~cm}$. Based on size, 17 cases were $<5 \mathrm{~cm}$ in the greatest dimension, 23 were $\geq 5 \mathrm{~cm}$ in size. 32 cases $(80.0 \%)$ were adenocarcinomas, 06 (15.0\%) mucinous adenocarcinomas and 02 cases $(5.0 \%)$ signet-ring cell carcinomas.

32 cases $(80 \%)$ were moderately differentiated and 8 $(20.0 \%)$ were poorly differentiated. 13 cases $(32.5 \%)$ were in stage pT2, $21(52.5 \%)$ in pT3 and $6(15.0 \%)$ in pT4. 17 cases $(42.5 \%)$ were N0, 13 cases $(32.5 \%)$ were N1, 8 cases $(20.0 \%)$ were N2 and 2 cases $(5.0 \%)$ were N3. Metastasis could not be ascertained. 
Out of 40 cases studied, $8(20 \%)$ showed perineural invasion. Amongst them, 6 cases were moderately differentiated and 2 were poorly differentiated. $16(40 \%)$ out of 40 cases were positive for LVI by H\&E. 27 (67.5\%) showed LVI with D2-40. 10 (25\%) showed both lymph node involvement and lymphatic infiltration on H\&E compared to $22(55 \%)$ on D2-40. D2-40 detected 11 (27.5\%) more cases with lymphatic invasion than H\&E. The difference between both the methods of assessing vascular invasion was significant by $\mathrm{McNemar}$ test $(\mathrm{P}=0.0127)$

D2-40 lymphatic microvascular counts ranged from 9 to 18 with a mean of $13.53 \pm 2.552 .27(67.5 \%)$ cases had moderate lymphatic microvascular density (LMVD), 10 (25\%) had high and $3(7.5 \%)$ had low LMVD. LVI detected by D2-40 correlated significantly with LMVD $(r=0.473, P$ $=0.002)$ and nodal status $(\mathrm{r}=0.663, \mathrm{P}=0.000003)$.
Lymphatic microvessel counts correlated significantly with nodal status $(\mathrm{r}=0.462, \mathrm{P}=0.003)$ and stage $(\mathrm{pT})(\mathrm{r}$ $=0.327, \mathrm{P}=0.040)$. LVI showed no significant correlation with stage $(\mathrm{pT})(\mathrm{P}=0.211)$, grade $(\mathrm{P}=0.623)$, size $(\mathrm{P}=$ $0.326)$ or perineural invasion $(\mathrm{P}=0.744)$. LMVD showed no significant correlation with grade $(\mathrm{P}=0.80)$, size $(\mathrm{P}=$ $0.381)$ or perineural invasion $(\mathrm{P}=0.804)$.

Peritumoral lymphatics were found to be present in 38 $(95 \%)$ and intratumoral lymphatics were present in 36 $(90 \%)$ cases. Intratumoral lymphatics showed significant correlation with nodal status $(\mathrm{r}=0.357, \mathrm{P}=0.024)$ but peritumoral lymphatics showed no such correlation $(\mathrm{P}=$ 0.127). Intratumoral and peritumoral lymphatics showed no significant correlation with tumor size $(\mathrm{P}=0.757, \mathrm{P}=$ $0.831)$, stage (pT) $(\mathrm{P}=0.325, \mathrm{P}=0.500)$, grade $(\mathrm{P}=0.304$, $\mathrm{P}=0.481)$ or perineural invasion $(\mathrm{P}=0.799, \mathrm{P}=0.48)$.
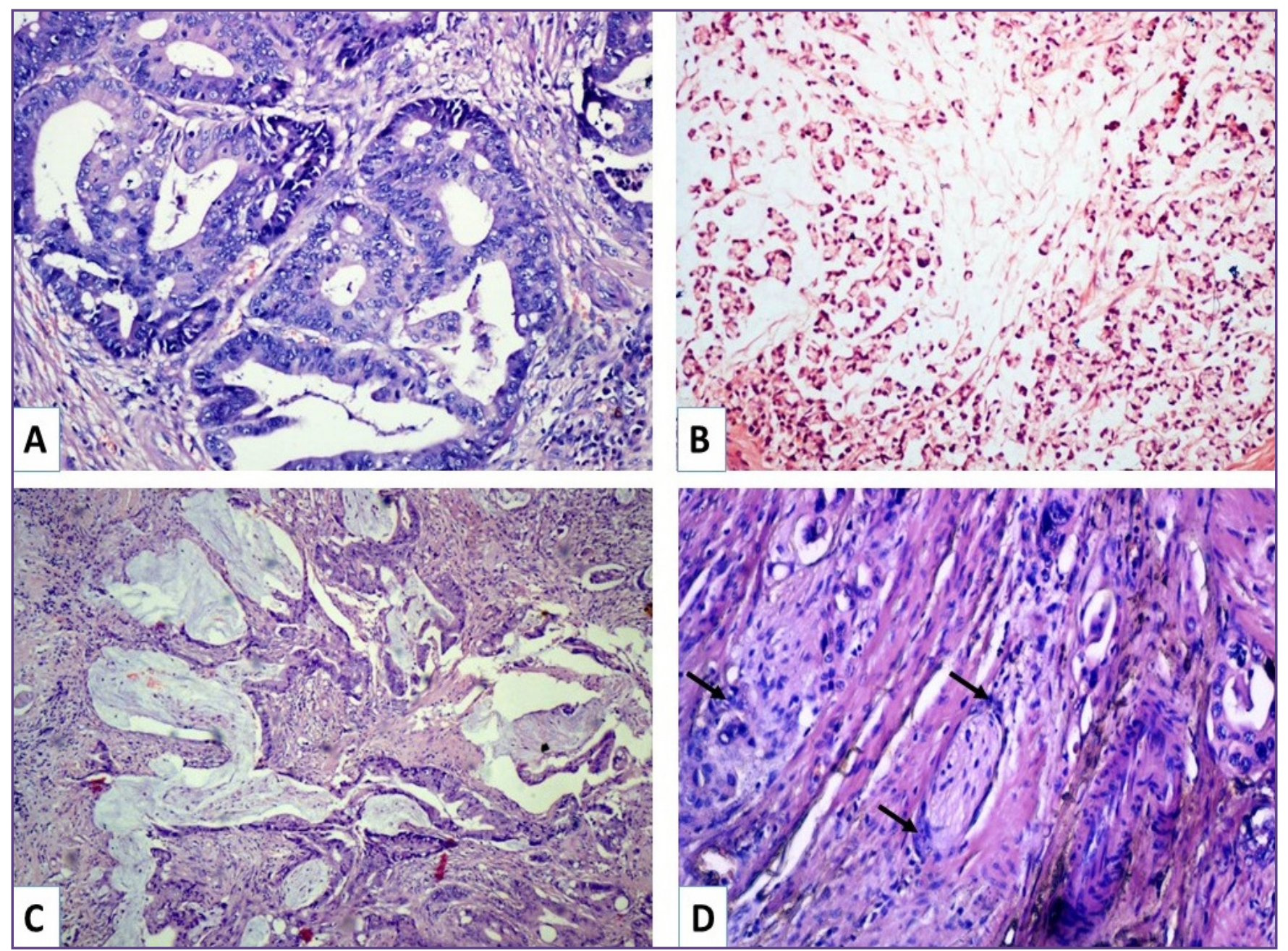

Fig. 1 (A): Adenocarcinoma colon. Grade moderately differentiated (H\&E, x100). (B): Signet ring cell carcinoma colon. Grade poorly differentiated (H\&E, x100). (C): Mucinous adenocarcinoma colon. Grade poorly differentiated (H\&E, x100). (D): A case of colorectal adenocarcinoma showing perineural invasion (H\&E, x100). 


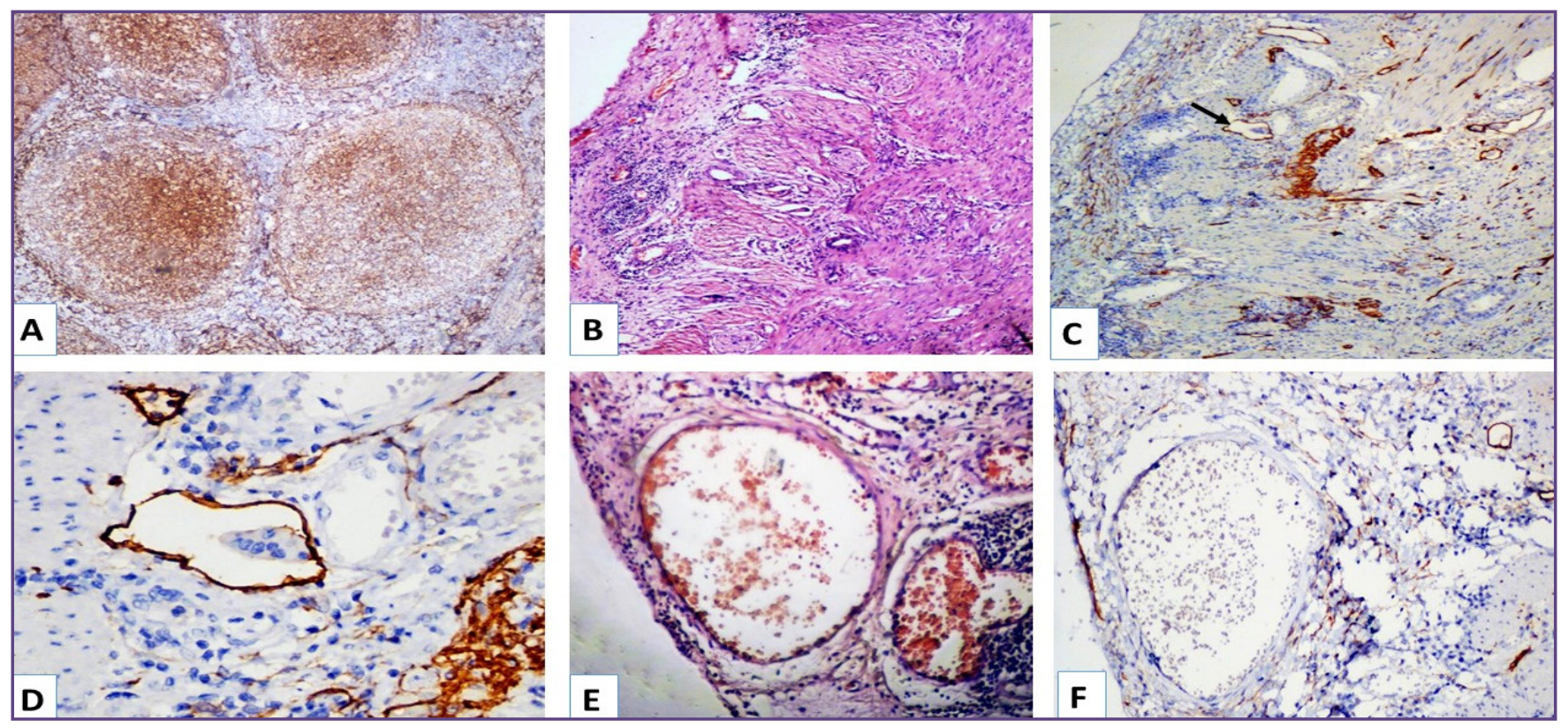

Fig. 2 (A): Section from tonsil taken as positive control showing immunostained lymphatics (D2-40, x40). (B): Section from colorectal carcinoma showing no apparent lymphovascular invasion (H\&E, X40). (C): Corresponding section showing lymphatic invasion (arrow) on immunostaining with D2-40 (x40). (D): Higher magnification of the same showing lymphovascular invasion with clearly outlined lymphatic endothelium on D2-40 (x200). (E): Blood vessel on H\&E (x100). (F): Blood vessel is not stained with D2-40 whereas surrounding area shows lymphatics stained with D2-40 (x100).

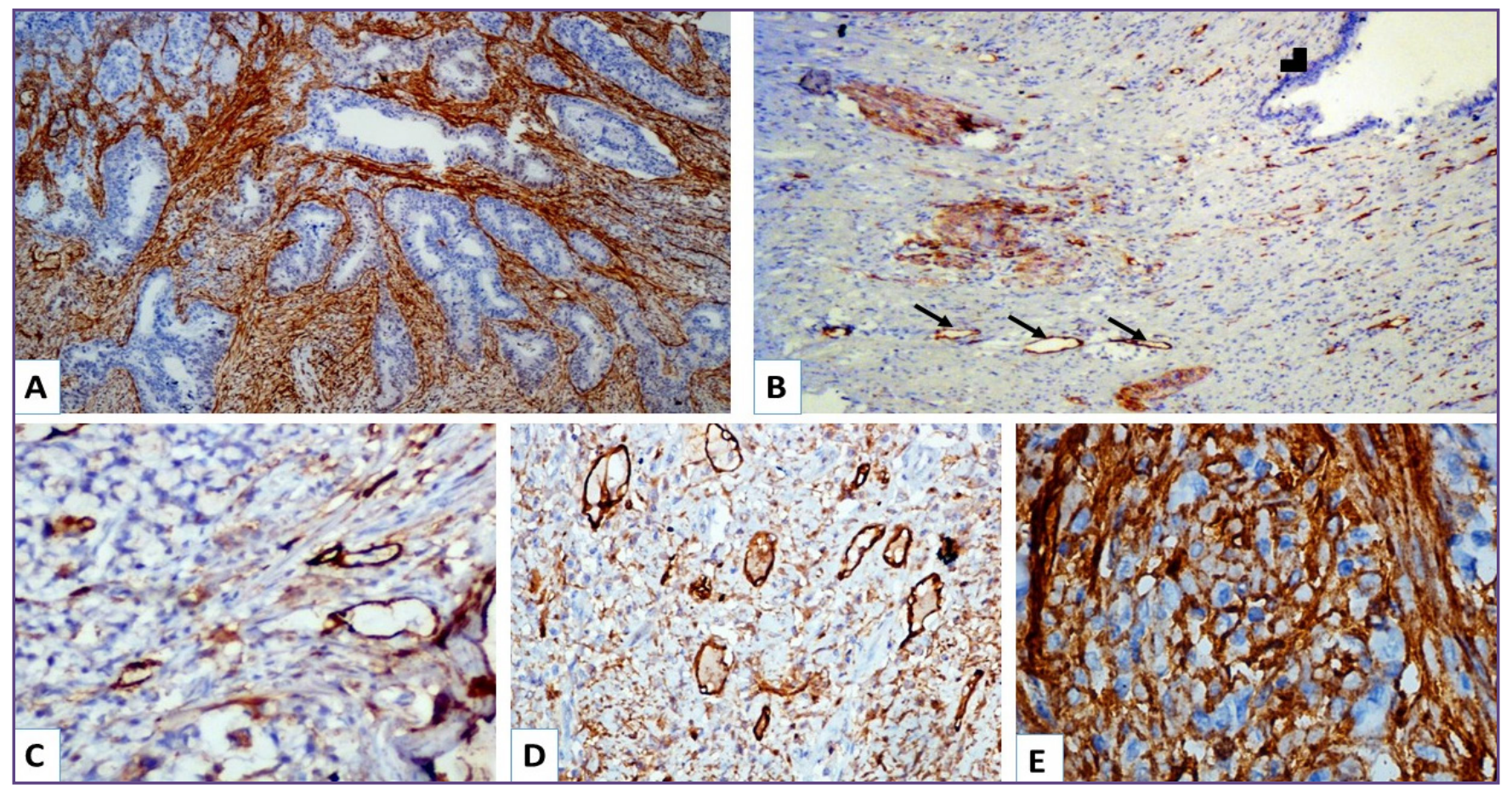

Fig. 3 (A): Intratumoral lymphatics (D2-40 immunostaining, x100). (B): Peritumoral lymphatics (arrows) away from a focus of tumor (arrowhead) (D2-40, x100). (C): A case with an area showing <10 lymphatic vessels. Such areas were taken as low lymphatic microvascular density. (D2-40 IHC, x400). (D): A case with areas having 10-15 lymphatic vessels which were taken as having moderate lymphatic microvascular density. (D2-40 IHC, x400). (E): An area from a different case having >15 lymphatic microvessels that was counted as an area with high lymphatic microvascular density. (D2-40 IHC, x400). 


\section{Discussion}

Colorectal cancer is one of the commonest causes of cancerrelated deaths. ${ }^{[9]}$ The lymphatics make up an important pathway of metastasis and extent of nodal involvement is an important prognostic factor. ${ }^{[8]}$

Lymphatic invasion is an important finding in assessing primary tumors. Some studies have found it as a predictor of survival. ${ }^{[10]}$ Studies have shown the prognostic value of lymphatic vascular density. ${ }^{[4]} \mathrm{D} 2-40$ is a specific marker for lymphatics to detect LVI and evaluate LMVD. ${ }^{[11],[12]}$ In the present study, 40 cases were analyzed for LVI and LMVD by $\mathrm{D} 2-40$ and their correlation with various parameters.

Lymphatic invasion: On H\&E, LVI is detected as tumor emboli in spaces lined by a single layer of endothelial cells. ${ }^{[11]}$ However, many difficulties arise in identifying lymphatic vessel walls by H\&E. One is in detecting tumor emboli when the lumen of a lymphatic vessel is obliterated, the other is the impossibility of distinguishing retraction artifacts during fixation from true tumor emboli floating in lymphovascular spaces. ${ }^{[6],[13]}$

Another problem with H\&E is inability to know whether the involved space is lymphatic or vascular. These problems result in missing lymphatic invasion, false positivity for lymphatic invasion or misdiagnosing an invasion in blood vessel as lymphatic invasion. To decrease this inaccuracy, D2-40 immunostaining was used.

In this study, LVI was detected in $11(27.5 \%)$ more cases by D2-40 immunostaining as compared to H\&E, which was significant $(\mathrm{P}=0.0127)$, similar to studies by Kawaura et $\mathrm{al}^{[13]} \&$ Walgenbach et $\mathrm{al}^{[14]}$.

This study showed a significant correlation between LVI on D2-40 and lymph node status and lymphatic microvascular density.

Lymphatic Microvascular Density: Studies have evaluated the correlation of lymphangiogenesis in colon carcinoma with prognostic parameters ${ }^{[15], ~[16]}$ but a few of them used D2-40 and observed a trend between lymphatic density and lymph node metastases, although not statistically significant. ${ }^{[15]}$ Saad et $\mathrm{al}^{5}$ demonstrated that using D2-40 and CD31, microvessel counts correlated significantly with the presence of LVI and nodal metastases.

Lymphatic microvascular counts ranged from 9 to 18 with a mean of $13.53 \pm 2.552$ in this study compared to Naik et $\mathrm{al}^{[17]}(17 \pm 9.7)$ and Saad et al ${ }^{[4]}(18 \pm 9)$.

This study showed significant correlation of LMVD with LVI, nodal metastases and pT stage similar to Saad ${ }^{[4]}$. Intratumoral lymphatics were found in $90 \%$ of cases which was similar to a study by Kuroyama. ${ }^{[15]}$ In this study, intratumoral lymphatics showed significant correlation with lymph node status $(\mathrm{P}=0.024)$. Peritumoral lymphatics were seen in $95 \%$ of the cases. Peritumoral lymphatics were enlarged and dilated and intratumoral lymphatics were small and flattened similar to other studies. [4], [18], [19], ${ }^{[20]} \mathrm{D} 2-40$ proved very useful in this study in overcoming the inaccuracy in identifying true LVI and evaluating LMVD, peritumoral and intratumoral lymphatics (which is not possible on H\&E) and their correlation with histopathological parameters.

This study also underlines the potential role of lymphangiogenesis and lymphatic invasion in tumor progression and lymph node metastasis.

\section{Conclusion}

Colorectal adenocarcinoma is one of the most common malignancies causing death worldwide. Lymph node status and lymphovascular invasion play an important role in the disease progression and outcome. 40 cases were studied for tumour morphology including the tumour size, location, histologic type, grade, pT stage, nodal status and immunohistochemistry was done with D2-40.

Immunostaining with D2-40 significantly increased the detection rate of LVI as compared to H\&E underlying the importance of D2-40 IHC as an investigative modality. In this study, LVI correlated significantly with nodal status suggesting its role in disease metastasis. LVI also showed significant correlation with LMVD.

LMVD was more in cases with high pT stage and lymph node status as compared to cases with lower pT stage and nodal status indicating the value of lymphangiogenesis in disease progression and metastasis. D2-40 has the potential as a marker in better understanding the pathogenesis related to lymphatics in the colorectal cancer cases as well as in the prognosis of the disease.

More studies are necessary to further evaluate and ascertain the prognostic value of lymphatic invasion and the induction of tumor lymphangiogenesis and its role in human cancer progression.

\section{References}

1. Parkin DM, Pisani P, Ferlay J. Global cancer statistics. CA Cancer J Clin. 1999;49(1):33-64.

2. Ferlay J, Shin HR, Bray F et al. Estimates of worldwide burden of cancer in 2008. Int J Cancer. 2010;127(12):2893-917.

3. Parums DV, Cordell JL, Micklem K et al. JC70: a new monoclonal antibody that detects vascular endothelium associated antigen on routinely processed tissue sections. J Clin Pathol. 1990;43:752-7. 
4. Saad RS, Kordunsky L, Liu YL et al. Lymphatic microvessel density as prognostic marker in colorectal cancer. Mod Pathol. 2006;19:1317-23.

5. Marks A, Sutherland DR, Bailey D et al. Characterization and distribution of an oncofetal antigen (M2A antigen) expressed in testicular germ cell tumors. $\mathrm{Br} \mathrm{J}$ Cancer. 1999;80:569-78.

6. Kahn HJ, Bailey D, Marks A. Monoclonal antibody, D240: A new marker of lymphatic endothelium, reacts with Kaposi's sarcoma and a subset of angiosarcomas. Mod Pathol. 2002;15:434-40.

7. Niakosari F, Kahn HJ, Marks A et al. Detection of lymphatic invasion in primary melanoma with monoclonal antibody D2-40: a new selective immunohistochemical marker of lymphatic endothelium. Arch Dermatol. 2005; 141:440-444

8. Maghraby HK, Elsarha AI, Saad RS. Peritumoral lymphatic vessel density as a prognostic parameter in endometrial carcinoma: An immunohistochemical Study. Indian J Pathol Microbiol. 2010;53(3):465-9

9. Stewart B, Wild C. Colorectal cancer. In: Bosman F, Hamilton S, Lambert R, editors. World cancer report. Lyon: IARC; 2014. P. 392-99

10. Compton CC. Pathology report in colon cancer : what is prognostically important? Dig Dis. 1999;17(2):67-79

11. Kahn HJ, Marks A. A new monoclonal antibody, D2-40, for detection of lymphatic invasion in primary tumors. Lab Invest. 2002;82(9):1255-7.

12. Fogt F, Zimmerman RL, Ross HM et al. Identification of lymphatic vessels in malignant, adenomatous and normal colonic mucosa using the novel immunostain D2-40. Oncol Rep 2004;11(1):47-50
13. Kawaura K, Fujii S, Murata $\mathrm{Y}$ et al. The Lymphatic Infiltration Identified by D2-40 Monoclonal Antibody Predicts Lymph Node Metastasis in Submucosal Invasive Colorectal Cancer. Pathobiology. 2007;74(6):328-35

14. Walgenbach-Bruenagel G, Tolba RH, Varnai AD et al. Detection of lymphatic invasion in early stage primary colorectal cancer with the monoclonal antibody D2-40. Eur Surg Res. 2006;38(5):438-44

15. Kuroyama S, Kobayashi N, Ohbu M et al. Enzyme histochemical analysis of lymphatic vessels in colon carcinoma: Occurrence of lymphangiogenesis within the tumor. Hepatogastroenterology. 2005;52(64):1057-61

16. Parr C, Jiang WG. Quanitative analysis of lymphangiogenic markers in human colorectal cancer. Int $\mathrm{J}$ Oncol. 2003;23(2):533-9

17. Naik VR, Jaafar H, Seng CE. Lymphatic channel density in colorectal adenocarcinoma. Indian J Pathol Microbiol. 2010;53(1):12-4.

18. Gombos Z, Xu X, Chu CS et al. Peritumoral lymphatic vessel density and vascular endothelial growth factor $\mathrm{C}$ expression in early stage squamous cell carcinoma of the uterine cervix. Clin Cancer Res. 2005;11(23):8367-71.

19. Sipos B, Klapper W, Kruse ML et al. Expression of lymphangiogenic factors and evidence of intratumoral lymphangiogenesis in pancreatic endocrine tumors. Am J Pathol. 2004;165(4):1187-97

20. Padera TP, Kadambi A, di Thomaso E et al. Lymphatic metastasis in the absence of functional intratumor lymphatics. Science. 2002;296(5574):1883-6.
*Corresponding author:

Dr. Sarvek Bajaj, 30-P RCC Area Model Town, Fatehabad, Haryana; INDIA; PIN: 125050

Phone: +919741860166

Email:drsarvek@gmail.com

Financial or other Competing Interests: None.
Date of Submission : 24.10.2016

Date of Acceptance : 15.01.2017

Date of Publication : 07.04.2017 